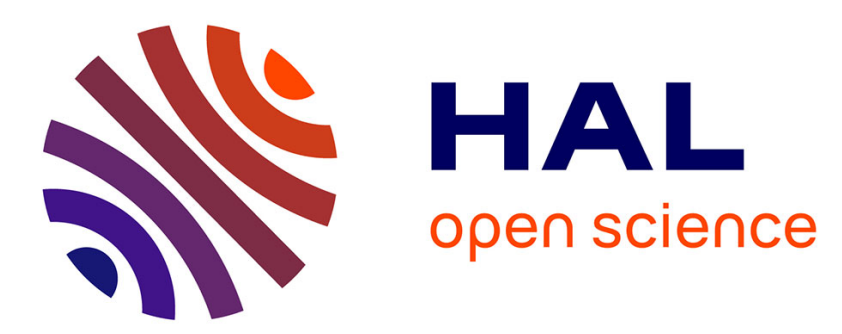

\title{
The impact of vaccine side effects on the natural history of immunization programmes: an imitation-game approach
}

\author{
Alberto d'Onofrio, Piero Manfredi, Piero Poletti
}

\section{- To cite this version:}

Alberto d'Onofrio, Piero Manfredi, Piero Poletti. The impact of vaccine side effects on the natural history of immunization programmes: an imitation-game approach. Journal of Theoretical Biology, 2011, 273 (1), pp.63. 10.1016/j.jtbi.2010.12.029 . hal-00669204

\section{HAL Id: hal-00669204 https://hal.science/hal-00669204}

Submitted on 12 Feb 2012

HAL is a multi-disciplinary open access archive for the deposit and dissemination of scientific research documents, whether they are published or not. The documents may come from teaching and research institutions in France or abroad, or from public or private research centers.
L'archive ouverte pluridisciplinaire HAL, est destinée au dépôt et à la diffusion de documents scientifiques de niveau recherche, publiés ou non, émanant des établissements d'enseignement et de recherche français ou étrangers, des laboratoires publics ou privés. 


\section{Author's Accepted Manuscript}

The impact of vaccine side effects on the natural history of immunization programmes: an imitationgame approach

Alberto d'Onofrio, Piero Manfredi, Piero Poletti

PII: $\quad$ S0022-5193(10)00684-3

DOI: $\quad$ doi:10.1016/j.jtbi.2010.12.029

Reference: $\quad$ YJTBI6298

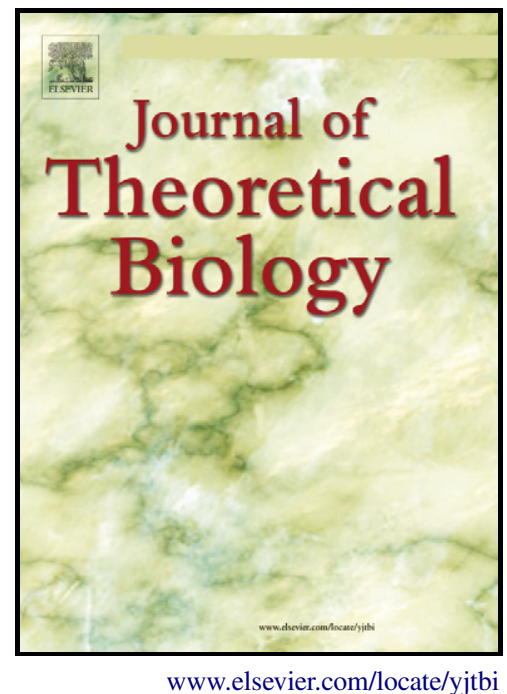

To appear in: $\quad$ Journal of Theoretical Biology

Received date: $\quad 8$ October 2010

Revised date: 20 December 2010

Accepted date: 20 December 2010

Cite this article as: Alberto d'Onofrio, Piero Manfredi and Piero Poletti, The impact of vaccine side effects on the natural history of immunization programmes: an imitation-game approach, Journal of Theoretical Biology, doi:10.1016/j.jtbi.2010.12.029

This is a PDF file of an unedited manuscript that has been accepted for publication. As a service to our customers we are providing this early version of the manuscript. The manuscript will undergo copyediting, typesetting, and review of the resulting galley proof before it is published in its final citable form. Please note that during the production process errors may be discovered which could affect the content, and all legal disclaimers that apply to the journal pertain. 


\title{
The impact of vaccine side effects on the natural history of immunization programmes: an imitation-game approach
}

\author{
Alberto d'Onofrio ${ }^{1 *}$ Piero Manfredi ${ }^{2}$ and Piero Poletti ${ }^{3,4}$
}

December 22, 2010

${ }^{1}$ Department of Experimental Oncology, European Institute of Oncology, Via Ripamonti 435, Milano, Italy

EMAIL: alberto.donofrio@ifom-ieo-campus.it

${ }^{2}$ Department of Statistics and Applied Mathematics, Universit di Pisa. Via Ridolfi 10, Pisa, ITALY. EMAIL: manfredi@ec.unipi.it

${ }^{3}$ Department of Mathematics, Universitá di Trento, Via Sommarive 14, Trento, Italy

${ }^{4}$ Predictive models for Biomedicine and Environment, Fondazione Bruno Kessler, Via Sommarive 18, Trento, Italy

EMAIL: poletti@fbk.eu

\begin{abstract}
When the incidence and prevalence of most common vaccine preventable childhood infectious diseases are constantly low, as is the case in many industrialized countries, the incidence of vaccine-associated side effects might become a key determinant in vaccine demand. We study an SIR transmission model with dynamic vaccine demand based on an imitation mechanism where the perceived risk of vaccination is modelled as a function of the incidence of vaccine side effects. The model shows some important differences compared to previous game dynamic models of vaccination, and allows noteworthy inferences as regards both the past and future lifetime of vaccination programmes. In particular it is suggested that a huge disproportion between the perceived risk of disease and vaccination is necessary in order to achieve high coverages. This disproportion is further increased in highly industrialised countries. Such considerations represent serious challenges for future vaccination programmes.
\end{abstract}

Keywords: Epidemiological models, vaccination choices, imitation dynamics, vaccine side effects.

\section{Introduction}

Although forms of exemption to vaccination have always existed [23], the "natural history" of vaccination programmes, as part of the historical pathway through which humankind progressively rids itself of infectious diseases, has always been pervaded by a high degree of optimism [5]. However, recently this optimistic view has increasingly been challenged: for example, opposition to the whole-cell pertussis vaccine [12], the thimerosal case [16], and the MMR scare [20] can be considered evidence that in industrialized countries, the success story of vaccination is feeding back on itself. This is the consequence of two different processes. On the one hand, the high degree of herd immunity achieved by decades of successful immunization programmes has reduced the incidence of many infections to negligible levels. On the other hand the large, and increasing, number of vaccines routinely administered every year yields steady flows of vaccine-associated side effects [26], [27]. In the US approximately

*Corresponding Author 
30,000 reports of Vaccine Adverse Events are notified annually, with 10-15\% classified as serious [4]. In such circumstances the perception of the public will likely rank the perceived risk of suffering a vaccine side effect (VSE) as much higher than the corresponding risk of infection $[1,2,6,7]$. A common example is poliomyelitis in industrialized countries. For example in Italy during 1980-2000 the number of vaccine-induced polio cases was three times higher than wild polio cases [15]. Under voluntary vaccination high degrees of herd immunity might therefore incentivise vaccination free riding [3], [21], i.e. the parents' decision not to vaccinate children after comparing the perceived risk of disease and the perceived risk of vaccine side effects $[1,2,6]$. Vaccination free riding $[3,10,13,1,2,6,7,9,21,22,11]$ makes eradication impossible (unless special contact structures are considered [19]) and triggers stable oscillations in the infection prevalence.

Previous studies on the impact of vaccination free riding on endemic infections have focused on scenarios where the vaccine demand is driven by the time changes in the perceived risk of disease, measured through the current (or past) infection prevalence. Based on the above-cited literature on VSEs we instead suggest that in industrialized countries, where the incidence of common vaccine preventable infections is very low, the available information on vaccine side effects might become the main driving force of vaccine demand. The only paper devoted to this issue is [9], where, however, vaccine demand was phenomenologically modelled as a decreasing function of the perceived risk of suffering a vaccine-associated side effect. The perceived risk of VSEs was in its turn evaluated by the public from available information on current and past trends of side effects attributed to the vaccine. In this paper we shift the focus onto the impact of VSEs on the dynamics of immunization programmes for endemic infections, using an SIR transmission model with voluntary vaccination choice. Vaccination choices are described by an evolutionary game as in [2], where the vaccine uptake $p(t)$ is determined by an imitation process between agents (the parents of the children to be vaccinated) who are divided into "vaccinators" and "non-vaccinators". However, in [2] the perceived payoff of the "non-vaccinators" is proportional to the prevalence of the disease, whereas the payoff of "vaccinators" is constant, i.e. independent of VSEs.

The novelty of the present work is that the payoff of "vaccinators" is proportional to the incidence of vaccine-associated side effects. In turn, this incidence is assumed to be proportional to the actual vaccine uptake. We believe that this model represents a more appropriate description of the future evolution of immunization programmes in voluntary vaccination regimes. Indeed, the fact that available information on vaccine side effects might become the main driving force of vaccine demand is strongly supported by the empirical evidence, e.g. the case of England and Wales, where due to the MMR scare [20], first dose measles uptake fell for several years from $94 \%$ to about $75 \%$. Compared to [2] we also expand our basic model to include (a) nonlinear perceived costs of infection; (b) the possibility that the perceived costs of infection and vaccination are evaluated by the public using past values of state variables, for example due to information delay [7] or to the perception of long-term vaccine side effects [9].

\section{Materials and Methods}

\subsection{Dynamic vaccine demand and vaccine side effects}

We consider a family of models of the spread of a non-fatal SIR infection controlled by voluntary vaccination with a "perfect" vaccine administered in a single dose at birth and giving life-long immunity:

$$
\begin{aligned}
S^{\prime} & =\mu(1-p)-\mu S-\beta S I \\
I^{\prime} & =\beta S I-(\mu+\nu) I \\
p^{\prime} & =k_{1} \Delta E p(1-p)
\end{aligned}
$$

where: $S, I$ are the susceptible and infective fractions, and $p$ the vaccinated proportion among newborn children; $\beta>0, \mu>0, \nu>0$ denote the transmission, the mortality and the recovery rates. We assume that, in absence of vaccination, the disease is endemic, i.e. $R_{0}=\beta /(\mu+\nu)>1$.

The dynamics of $p$ obeys a learning by imitation [14] process where $k_{1}$ is the "imitation" coefficient 
and switching between the decisions to vaccinate or not to vaccinate, is determined by the payoff gain $\Delta E(t)$. The latter is given by the difference between the perceived payoff of vaccinators $-\rho_{V}(t)$, where $\rho_{V}(t)$ is the perceived risk of suffering a VSE, and the perceived payoff of non-vaccinators: $-\rho_{I}(t)$, where $\rho_{I}(t)$ is the perceived risk of suffering serious illness due to infection.

We note that, irrespective of the specific forms of $\Delta E(t)$, the family of models (1-2-3) has the following three equilibria: (i) an unstable disease-free equilibrium with no vaccinators $A=(1,0,0)$; (ii) a pure-vaccinator disease-free equilibrium $B=(0,0,1)$; (iii) the pre-vaccination endemic equilibrium $C=\left(S_{S I R}=R_{0}^{-1}, I_{S I R}=\mu\left(1-R_{0}^{-1}\right) /(\mu+\nu), 0\right)$.

The stability of $B$ and $C$ and the existence of further equilibria depend on the specific types of the payoff gain.

Modelling of the dynamics of $p$ by an imitation game was introduced by Bauch in the seminal paper [2], where a specific model of the family (1-2-3) was proposed, where: i) the perceived payoff of vaccinators is constant: $-\rho_{V}=-r_{V}$; and $\left.i i\right)$ the perceived payoff of non-vaccinators is proportional to the infective prevalence $I(t):-\rho_{I}(t)=-r_{I} m I(t)$, where $m I(t)$ is an estimate of the current risk of infection, and $r_{I}$ is the risk of serious disease as a consequence of infection. Hence:

$$
\Delta E(t)=r_{I} m I(t)-r_{V}=r_{V}(\vartheta I(t)-1),
$$

where $\vartheta=m r_{I} / r_{V}$ is proportional to the relative cost of the non-vaccinator strategy.

In Bauch's model the $B$ equilibrium is unstable and there is a fourth equilibrium: the post-vaccination equilibrium $D=\left(R_{0}^{-1}, \vartheta^{-1}, \widehat{p}\right)$ where: $\widehat{p}=(1+\nu / \mu)\left(I_{S I R}-\vartheta^{-1}\right)$. At $\vartheta=\vartheta_{0}=I_{S I R}^{-1}$ there is a transcritical bifurcation between $C$ and $D$. In turn, the stability/instability of $D$ depends on the product $k_{1}\left(\vartheta-\vartheta_{0}\right)$.

The assumption in [2] that the perceived risk of VSEs $\rho_{V}(t)$ is constant can be justified if the public correctly evaluates this risk as the ratio between total VSEs per unit time, given by $\alpha(\mu N) p(t)$ $(\alpha \in(0,1))$ where $N$ is the total population size (so that $\mu N$ is the yearly number of births), and $\alpha$ the per-capita probability of incurring a VSE during a single vaccine administration, and the total number of vaccinations administered for that specific disease $\mu N p(t)$. We instead suppose that the public evaluates the risk of VSEs by using the available information on the total number of vaccineassociated side effects, or, which is equivalent, on the ratio between the total number of vaccine associated side effects and the total number of newborn children per unit of time. We therefore set:

$$
\rho_{V}(t)=\alpha p(t)
$$

The implication of (4) is that periods of large vaccine uptake negatively feed back, through an increase in the incidence of VSEs, into the proportion of parents favourable to vaccination.

We model the perceived risk of infection as an increasing function $h_{1}(\cdot) \geq 0$ of the information index $M$, introduced in $[6,7]$, that summarizes the publicly available information on the infection:

$$
\rho_{I}(t)=h_{1}(M(t)) .
$$

The index $M$, used by agents to evaluate the risk of infection, may model not only current, but also past information. The case $h_{1}(0)>0$ accounts for the scenario where the disease is locally eliminated but disease re-emergence by external reintroduction is feared. In the simplest case, the information index $M(t)$ is related to the current prevalence of the infection as follows:

$$
M(t)=h_{2}(I(t)),
$$

where $h_{2}(\cdot)>0$ and $h_{2}^{\prime}(\cdot)>0$. Using (4)-(5)-(6) the dynamics of $p$ can be written as follows:

$$
p^{\prime}=k(h(I)-p) p(1-p)
$$

where $h(I)=\alpha^{-1} h_{1}\left(h_{2}(I)\right), k=\alpha k_{1}$.

First, note that from: $p^{\prime} \geq k(h(0)-p) p(1-p)$ it follows that $h(0) \geq 1 \Rightarrow p(t) \rightarrow 1$, so that we shall not consider this trivial case. On the contrary, if $h(0)<1$ then $p(t) \geq h(0)$ holds asymptotically, i.e. the collective coverage will at least reach the minimal level $h(0)$. Symmetrically, inequality 
$p^{\prime} \leq k h^{\operatorname{Max}} p(1-p)$ means that $p(t)$ is bounded by a logistic dynamics with kinetics constant $k h^{\text {Max }}$. Finally, if imitation dynamics is faster than the infection time scale: $k>>(\mu+\nu)^{-1}$, then $p(t)$ is at quasi-equilibrium:

$$
p(t) \approx \min (h(M(t)), 1)
$$

which is the phenomenological relationship first proposed in [6].

\subsection{The importance of time delays}

The assumption that $\Delta E$ depends only on current values of $I$ and $p$ is an approximation. For example agents might perceive that vaccines are responsible for VSEs arising with long time delays [9], as might be the case with auto-immune diseases [18]. Moreover, delays of different nature, concerning the information on the spread of the disease, may involve both $p(t)$ and $M(t)$ [6]. Thus in the case in which the perceived risk of vaccination is evaluated on past VSEs:

$$
\rho_{V}(t)=\int_{-\infty}^{t} Q_{H}\left(T_{v}\right) \alpha p\left(t-T_{v}\right) d T_{v}
$$

where $Q_{H}$ is a delay kernel. An important kernel is the exponentially fading memory: $Q_{H}(x)=$ $b \exp (-b x), b>0$, which allows reduction to ODE since it holds that:

$$
\rho_{V}^{\prime}=b\left(\alpha p(t)-\rho_{V}\right) \text {. }
$$

Scenarios such as long-term VSEs may require different kernels, more concentrated on past periods, such as the Erlang functions: $A_{n} x^{n-1} \exp (-b x)$, also allowing reduction to ODEs.

\section{Results}

\subsection{Endemic equilibria and their stability}

In this section we shall investigate the model (1)-(2)-(7) where no delays are present. Considering a generic $h(I)$, the system (1)-(2)-(7) has three equilibrium points $A$ (unstable), $B$ (unstable) and $C$, which, unlike [2], is always unstable since the linearized equation for $p$ reads $\eta^{\prime}=k h\left(I_{S I R}\right) \eta$. Moreover, two further equilibria are induced by the specific $\Delta E$ we introduced:

- A disease-free equilibrium with positive vaccine uptake $E_{d f e}=(1-h(0), 0, h(0))$.

- A new behaviour-related endemic equilibrium:

$$
E_{b e h}=\left(R_{0}^{-1}, I_{e}, h\left(I_{e}\right)\right),
$$

where $I_{e}$ is the unique solution of the equation:

$$
h(I)=1-R_{0}^{-1}-\frac{\mu+\nu}{\mu} I .
$$

Remark 3.1 The equilibrium $E_{\text {beh }}$ is equal to the endemic equilibrium of the model studied in [9], where, however, $p$ is not a state variable.

Since all equilibria are independent of $k$, which is an epidemiologically relevant parameter (we recall that it is proportional to the imitation coefficient $k_{1}$ determining the time-scales of the imitation phenomenon), this makes it natural to choose $k$ as a bifurcation parameter. Our main results (demonstrated in the Appendix) are as follows:

A) If

$$
\beta I_{e} \mu h^{\prime}\left(I_{e}\right)<\left(\mu+\beta I_{e}\right)\left(\left(\mu+\beta I_{e}\right)+2 \sqrt{\beta I_{e}(\mu+\nu)}\right)
$$

then $E_{b e h}$ is locally asymptotically stable (LAS) irrespective of the imitation speed $k$; 
B) On the contrary, if

$$
\beta I_{e} \mu h^{\prime}\left(I_{e}\right)>\left(\mu+\beta I_{e}\right)\left(\left(\mu+\beta I_{e}\right)+2 \sqrt{\beta I_{e}(\mu+\nu)}\right)
$$

holds then there are two positive values $k_{1}$ and $k_{2}>k_{1}$ such that:

1. If $0<k<k_{1}$ or $k>k_{2}$ then $E_{b e h}$ is LAS;

2. At $k=k_{1}$ and at $k=k_{2}$ there are Hopf bifurcations;

3. If $k \in\left(k_{1}, k_{2}\right)$ then $E_{b e h}$ is unstable.

C) In the case $k \in\left(k_{1}, k_{2}\right)$ the orbits $x(t)=(S(t), I(t), p(t))$ are oscillatory in the sense of Yabucovich [25]. This intuitively means that for sufficiently large $t$ all state variables are permanently oscillating, with regular or irregular oscillations. Formally, for $j=1,2,3$ it holds that:

$$
\operatorname{minlim}_{t \rightarrow+\infty} x_{j}(t)<\operatorname{maxlim}_{t \rightarrow+\infty} x_{j}(t) .
$$

D) If $h_{0}>p_{c}$ then $E_{d f e}$ is globally asymptotically stable (GAS); if $h_{0}<p_{c}$ then $E_{d f e}$ is unstable.

Remark 3.2 Note that, on dividing both the r.h.s. and the l.h.s. of (13) by $\beta \mu I_{e}$, after trivial calculations we obtain that to have Hopf bifurcation at $E_{b e h}$ the derivative of $h$ at $I_{e}$ has to be at least of order $O(\sqrt{\nu / \mu})$.

Remark 3.3 Property $\boldsymbol{D}$ shows that, unlike [2], elimination is possible in our model, although only if in absence of the disease the vaccinator payoff is so large as to yield a vaccine uptake greater than the elimination threshold.

To better understand the phenomenon of epidemic oscillations driven by vaccination choice we compare the above results with the recent literature on this subject. Unlike [2] where large values of $k\left(\vartheta-\vartheta_{0}\right)$ induce sustained oscillations around the endemic state, in our model oscillations are possible in an intermediate window of values of the imitation coefficient $k$. This means that both slow and fast imitation are stabilizing forces. Moreover, we note that the stability condition (12) has the same formal structure as that of the delayed model in [6], where dependence of the current vaccine uptake on past prevalence of the infection was required to obtain oscillations. Indeed, imitation is a (nonlinear) adjustment process introducing a delay, whose characteristic time scales are determined by the speed with which the vaccine uptake reacts to changes in the payoff gain. Heuristically, close to the equilibrium eq. (7) becomes:

$$
p^{\prime} \approx k h\left(I_{e}\right)\left(1-h\left(I_{e}\right)\right)(h(I)-p)=\Psi(h(I)-p)
$$

which may be read as an exponentially fading memory mechanism with average delay $1 / \Psi$. This suggests a route to estimate the elusive imitation coefficient $\mathrm{k}$, by preliminarily estimating the average delay $1 / \Psi$.

Finally, we note that the Yabucovitch oscillatority is a global result, unlike the Hopf bifurcation theorem, which is local. Although the nature (periodic, quasi-periodic or chaotic) of Yabucovitch oscillations cannot be determined a priori, this makes the predictions of our model very general.

Finally, it is easy to show that the inclusion of time delays does not affect the location and stability of equilibria $A, B, C$ and $E_{d f e}$, whereas the stability of $E_{b e h}$ may be affected, as seen in the numerical simulations below (see also the Appendix).

\subsection{Analysis of selected subcases}

We report numerical illustrations of noteworthy sub-cases of epidemiological interest. The basic reproduction number $R_{0}$ is set to 10 ; the recovery rate is set to either $\nu=0.1$ days $^{-1}$ (pertussis) or $\nu=(1 / 7)$ days $^{-1}$ (measles), which correspond to an average duration of the infectious period of respectively 10 days (pertussis) and one week (measles). Finally the life expectancy $L=1 / \mu$ is fixed either at $L=50$ years (used in [2] to more closely reproduce UK pertussis data) or at $L=75$ years, which is more representative of mortality in modern industrialized countries. 


\subsubsection{The basic unlagged model: the case of linear $h(I)$}

Letting $h(I)=\vartheta I$, the infection prevalence at $E_{b e h}$ is:

$$
I_{e}=I_{e}(\vartheta)=\frac{1-\frac{1}{R_{0}}}{1+\frac{\nu}{\mu}+\vartheta}=I_{S I R} \frac{1+\frac{\nu}{\mu}}{1+\frac{\nu}{\mu}+\vartheta}
$$

and the vaccine uptake is:

$$
p_{e}(\vartheta)=\vartheta I_{e}(\vartheta)=p_{c} \frac{\vartheta}{1+\frac{\nu}{\mu}+\vartheta}
$$

As regards the stability of $E_{b e h}$, it is of interest to assess the relative role of the two main behavioral parameters introduced by our model: $\vartheta$ and $k$. By applying (13) it is easy to show that a $\vartheta^{*}$ exists such that for $\vartheta>\vartheta^{*}$ then (13) is fulfilled and the two branches $k_{1}(\vartheta)$ and $k_{2}(\vartheta)$ of the bifurcation curve in the space $(k, \vartheta)$ exist and are analytically computable. Below $\vartheta^{*}$ the equilibrium $E_{b e h}$ is (at least) locally stable.

For example in the case $\nu=0.1$ days $^{-1}, \mu=1 / 50$ years $^{-1}$ it is $\left(\vartheta^{*}, k^{*}\right) \approx(280,0.042)$, from which the two branches $k_{1}(\vartheta)$ and $k_{2}(\vartheta)$ of the Hopf curve depart. For $\vartheta=1000$ (implying $I_{e}=3.18 \times 10^{-4}$ and $p_{e}=0.32$ ) we obtain $k_{2}^{-1}=4.75$ days and $k_{1}^{-1}=312$ days a range difficult to interpret. However, the heuristic average imitation delay $1 / \Psi$ correspondingly ranges between three weeks and about four years, close to the values found in [6]. The bifurcation curve in the $(k, \vartheta)$ and $\left(k^{-1}, \vartheta\right)$ parametric spaces are shown in Fig. 1.

\subsubsection{The basic unlagged model: the case of linear-affine $h(I)$}

The case $h(I)=\vartheta_{0}+\vartheta I$ is of interest since it assumes that even in scenarios of zero prevalence agents perceive a positive risk $\vartheta_{0}$ of infection re-emergence. We get:

$$
I_{e}(\vartheta)=\frac{1-R_{0}^{-1}-\vartheta_{0}}{1+\frac{\nu}{\mu}+\vartheta}, p_{e}(\vartheta)=\vartheta_{0}+\vartheta \frac{1-R_{0}^{-1}-\vartheta_{0}}{1+\frac{\nu}{\mu}+\vartheta}
$$

Thus the perception of such a risk has a positive effect on equilibrium uptake. The dependence of the bifurcation curve on $\vartheta_{0}$ may be non-monotone, as in the right-hand panel of Fig. 1.

\section{Substantive implications of vaccine side effects for vaccina- tion programmes}

\subsection{The epidemiological transition and vaccination payoff}

We use equilibrium results from the simple case of linear $h(I)$ to explore the impact of human progress on the natural history of vaccination programmes. Note that in the parametric set we are considering the dimensionless quantity $\nu / \mu$ ranges from about 1826 (pertussis in a population with low lifeexpectancy) up to about 3913 (measles in a population with high life-expectancy). Thus, achieving a large equilibrium uptake requires very large $\vartheta$, i.e. of the order of $\nu / \mu$. Since $\vartheta=r_{I} m / \alpha$, it follows that to achieve large equilibrium uptakes for measles or pertussis, the perceived cost of serious disease has to be at least three order of magnitude higher than the perceived cost of VSE. Though this seems surprising, we feel it is consistent with what was still observed at the beginning of the 20th century, when the risk of serious sequelae following measles or scarlet fever was extremely large (100250 deaths per 100000 cases of disease), and the absence of vaccines was keeping the risk of infection very high. In such circumstances even a large probability of suffering a VSE from a vaccine could have been tolerated by the community. This also suggests that industrialized countries could face serious difficulties in maintaining high vaccine uptake in the future. Indeed, completion of the epidemiological transition [24], i.e. the historic change in the cause composition of mortality in industrialized countries from infectious and nutritional diseases to chronicdegenerative ones, led to a dramatic fall in the levels of serious morbidity and mortality 
from all infectious diseases. Together with the current higher-than-ever degrees of herd immunity allowed by decades of sustained vaccination, this has reduced the perceived risks of serious disease from most infections to negligible levels. Finally, as regards the role played by life expectancy on the steady state $E_{b e h}$, our results suggest that the achievement of a given equilibrium uptake in industrialized countries (i.e. with a very high life expectancy) requires a much larger value of $\vartheta$ compared to developing countries. This is consistent with the fact that in regimes with low mortality (also as a consequence of vaccinations), agents demand a vaccine only if the relative risks of suffering a VSE are very low. We stress that our predictions are equilibrium ones based on a simple deterministic model with homogeneous mixing. Nonetheless more realistic models would not substantially affect them.

\subsection{Simulations}

In our simulations we set $h(I)=\vartheta I$ and, to better emphasize our main messages, we allow immigration of infectives, according to two different hypotheses: a) a small constant transfer Imm from the susceptible to the infective state, representing a steady flow of infections as a consequence of international travelling; b) a few new infections appear at some stage once and for all, in order to mimic the possibility of infection resurgence. Indeed, in the oscillatory regime the infection prevalence $I(t)$ may reach extremely low values, which calls into question the appropriateness of a deterministic model. This drawback is avoided by assumption a, also used in [2]. Imm is set to one infective individual per week in a population of $5 \times 10^{6}$ individuals.

\subsubsection{The basic unlagged model}

We first assess the impact of VSEs on the transient infection dynamics triggered by a new vaccination introduced at the pre-vaccination endemic state, and under assumption $\mathbf{a}$. The vaccine is introduced at time $t=0$, with initial vaccine uptake set to $0.95>p_{c}$. Vaccine side effects occur from the beginning of the programme. We set $\vartheta=15000$ (implying $p_{e} \approx 0.71, k=0.002$ days $^{-1}$ and $1 / \Psi \approx 7$ years). Note that $k$ is small since it is the product between the natural imitation rate and the low probability $\alpha$ of suffering a VSE. As predicted by (13), the system converges, in epidemiologically reasonable time scales, to a stable limit cycle. With reference to Fig. 2, the vaccine uptake (right-hand panel) starts declining soon after the programme starts, due to the onset of VSEs, and falls below the critical threshold $p_{c}$ in less than four years. Thus the effective reproduction number $R_{E}(t)=R_{0} * S(t)$ (lefthand panel) initially declines but then increases and exceeds the unit threshold at $t \approx 8$ years, yielding a new epidemic outbreak at $t \approx 10$ years. During this rather long 'honey-moon' period the circulation of the infection is essentially sustained by immigration. Note that $p(t)>p_{c}$ for a rather long period of time (about 36 per cent of total time). During such periods routine vaccination surveys would reveal a satisfactorily high coverage. Therefore they could not explain the endemic persistence of infection. The role played by $k$ is illustrated in Fig. 3, which considers the values: $k=0.0005$ days $^{-1}, k=$ 0.002 days $^{-1}$ (as before), $k=0.0035$ days $^{-1}$, and which shows that: $\left.i\right)$ the average uptake is not significantly affected by $k$ and remains close to $\left.p_{e} ; i i\right)$ both the amplitude of oscillations of $p(t)$ and the fraction of total time where $p(t)>p_{c}$ increase in $k$; iii) the duration of the period between the start of the programme and a new epidemic outbreak is decreasing in $k$. For example, the 'low' $k$ value $\left(k=0.0005\right.$ days $\left.^{-1}\right)$ yields oscillations that are of small amplitude and that for a small portion of their period are such that $p(t)>p_{c}$. Moreover, there is an interval of 17 years about before a new epidemics, which is only a few years long for $k=0.0035$ days $^{-1}$. This is the consequence of the slow spread of information occurring for low $k$, which slows down the reactivity of the vaccine uptake to changes in the payoff gain. Note that if no external infections are introduced, then for small $k$ the infection prevalence is close to zero. This suggests that oscillations might produce stochastic elimination of infection.

We now study the introduction of a new vaccine for which no vaccine side effects are initially known. In this case it seems reasonable to assume that an intermediate period might exist during which no perception of VSEs arises. We consider $\alpha=0$ for $t<t_{1}$ where $t_{1}=10$ years, and that the starting point is the pre-vaccination steady state. Of course, the vaccine uptake start increasing since the payoff 
vaccination is initially positive $(\alpha=0)$. If VSEs raise after a very long time, or no VSEs are reported, then stochastic elimination is possible. By contrast, if evidence of VSEs emerges, the scenario reduces straightaway to the cases treated above. If the imitation process is very slow, VSEs appear before reaching a sufficient coverage and a sub-optimal vaccination coverage is achieved (left-hand panel of Fig. 4). Instead for a larger value of $k$, the infective fraction is very close to 0 such that stochastic elimination may occur (central panel of Fig. 4). Finally, for an intermediate range of $k$ there is onset of oscillations, but at the end of their transitory the minimum $I(t)$ is very close to 0 and elimination can again occur (right-hand panel of Fig. 4).

Finally, we simulated the case where eradication was achieved thanks to a period of compulsory vaccination, after which vaccination becomes voluntary. In this case VSEs induce individuals to switch to the 'non-vaccinator' strategy, thereby producing a decrease in vaccination coverage. This in turn increases the probability of infection re-emergence from imported cases. If a few new external infections are introduced (assumption b) before $R_{E}(t)=1$, then the occurrence or not of stochastic transient elimination depends again on $k$, with patterns similar to the previous case (see Fig. 5.a,5.b). Moreover, if individuals take into account a non zero risk of infection re-emergence from importation (the linear affine case $h(I)=\vartheta_{0}+\vartheta I$ ), the population is more 'protected' and the impact of the external infections can be reduced also for large values of $k$ (see Fig. 5.c).

\subsubsection{Impact of information-related delays}

An exponentially distributed lag in the $p$ term yields a model given by (1)-(2) complemented by:

$$
\left.p^{\prime}=k(h(I)-H)\right) p(1-p)
$$

and by (10). Fig. 6 reports the stability regions in the $(k, b)$ plane for $\vartheta=260, \vartheta \approx 279.5$ (which is close to the critical value $\vartheta^{*}$ in absence of delays) and $\vartheta=500$. Note that for $\vartheta=500>\vartheta^{*}$ for all $b$ there are two values $k_{l}(b ; \vartheta)$ and $k_{r}(b ; \vartheta)$ such that for $k_{l}(b ; \vartheta)<k<k_{r}(b ; \vartheta)$ the endemic state $E_{b e h}$ is unstable and it is also an easy matter to show that Yabucovitch oscillations can arise. Similar plots are obtained in all cases where $\vartheta>\vartheta^{*}$. This can be easily explained since for very large $b$ the lagged model reduces to the unlagged one that, for $\vartheta>\vartheta^{*}$, has an instability interval.

Interestingly, the right panel of 6 for $\vartheta=260<\vartheta^{*}$ shows instability regions that are uniquely due to the information delay, because in absence of the delay (i.e. for large $b$ ) there is no instability, as shown in the previous sub-sections. This confirms that the addition of time delays in the $p$ term (mimicking delayed onset of VSEs or delayed information acquisition) cumulates with the imitation delay in triggering instabilities. Fig. 7 reports the time course of vaccine uptake $p(t)$ for $\vartheta=260$ for two distinct cases. In the left-hand panel the behaviour in absence of delay is reported, showing convergence to the endemic state. The right-hand panel reports the behaviour for a delay of 250 days $\left(b=0.004\right.$ days $\left.^{-1}\right)$ in the occurrence of side effects, showing instead convergence to a limit cycle.

Let us now consider an exponentially fading memory in the perceived risk of infection: let $M$ stand for past prevalence, and $a$ the corresponding delaying rate. We set $h_{1}(M)=\vartheta M$, and $h_{2}(I)=I$. In Fig. 8 we plotted the stability regions in the $(k, a)$ plane for $\vartheta=260, \vartheta \approx 279.5$ (which is close to the critical value $\vartheta^{*}$ ) and $\vartheta=500$.

Finally, in the case where exponentially fading memories appear both in the perceived risk of vaccination (with rate $b$ ) and the perceived risk of infection (with rate $a$ ), numerical simulations showed a wide pattern of periodic behaviour, including very long periods (an example with alternating low and high peaks is reported in the left-hand panel of Fig. 9), and also aperiodicity (as in the right-hand panel of Fig. 9)

\section{Concluding remarks}

We investigated an SIR transmission model with voluntary vaccination. Unlike [2] we consider a dynamic perceived risk of vaccination proportional to the trends of VSEs. Mathematical analysis of the model confirms already known results, such as the onset of behaviour-triggered steady oscillations about the post-vaccination endemic state. In addition it shows some noteworthy differences compared 
to findings elsewhere. For example global elimination may, or may not, be possible depending on the actual magnitude of the baseline perceived risk of disease relative to the risk of vaccine side effects. As regards the pre-vaccination equilibrium, differently from [2], it is always unstable, so that any vaccination programme will initially be successful, at least partially. Moreover oscillations occur, provided the relative risk of disease is large enough, in a bounded window of the imitation parameter. In addition, we also considered the impact of time delays on the vaccination payoff gain, which is a realistic feature. Finally, the model allows noteworthy inferences on the future lifetime of vaccination voluntary programmes. It suggests that in order to achieve high equilibrium uptakes for e.g. measles, the perceived cost of the disease must be at least three orders of magnitude higher than the perceived cost of vaccine-associated side effects, and moreover that this requirement is further increased under situations with large life expectancies. Both these facts seem to be consistent with real world observations, and suggest that maximal vaccination effort by international institutions should target increasing vaccine uptake in less developed countries where the risk of serious sequelae is still high, and life expectancy is still low. Simulations finally provide further interesting insights on the effect of behavioural parameters on vaccine uptake.

\section{Appendix}

The biomathematical properties listed in section 3 may be demonstrated as follows:

Property A Let us define, to simplify the notation, the parameter:

$$
\Psi=k h\left(I_{e}\right)\left(1-h\left(I_{e}\right)\right) .
$$

Linearizing at $E_{b e h}$ yields the following characteristic polynomial with positive coefficients:

$$
\lambda^{3}+\left(\Psi+\mu+\beta I_{e}\right) \lambda^{2}+\left(\left(\mu+\beta I_{e}\right) \Psi+(\mu+\nu) \beta I_{e}\right) \lambda+\beta I_{e}\left(\mu+\nu+\mu h^{\prime}\left(I_{e}\right)\right) \Psi
$$

for which the Routh-Hurwitz condition yields this inequality in the variable $\Psi$ :

$$
R H(\Psi)=\left(\mu+\beta I_{e}\right) \Psi^{2}+\left(\left(\mu+\beta I_{e}\right)^{2}-\beta I_{e} \mu h^{\prime}\left(I_{e}\right)\right) \Psi+\left(\mu+\beta I_{e}\right) \beta I_{e}(\mu+\nu)>0 .
$$

Since the coefficients of power 0 and 2 of eq. (19) are positive, and taking also into account the sign of the coefficient of power 1 , it is easy to show that if (12) holds then $E_{b e h}$ is LAS.

Property B If (13) holds then there are two positive values $0<k_{1}<k_{2}$ such that if $k<k_{1}$ or $k>k_{2}$ then $E_{b e h}$ is LAS; moreover, if $k_{1}<k<k_{2}$ then $E_{b e h}$ is unstable and $k_{1}$ and $k_{2}$ are Hopf points. After some algebra it is easy to verify that at the Hopf points there are Hopf bifurcations since the nonzero speed condition $\operatorname{Re}\left(\left.(d \lambda / d k)\right|_{\lambda= \pm i \omega_{\text {Hopf }}}\right) \neq 0$ is fulfilled.

Property C As regards the Yabucovitch oscillations, note that: i) the bounded set

$$
A=\left\{(S, I, p) \in R_{+} \mid 0 \leq S+I \leq 1-h(0), h(0) \leq p \leq 1\right\}
$$

is positively invariant and attractive; ii) $E_{b e h}$ is unstable, and $E_{d f e}$, which is in the boundary of $A$, has as the stable manifold the line $(w, 0,1) w \in[0,1]$ to which $E_{b e h}$ does not belong, excluding heterocline orbits. Thus we may apply the Yabucovitch theorem [25].

Property D Since asymptotically $p(t) \geq h(0)$, it follows that for large times:

$$
S^{\prime} \leq \mu(1-h(0)-S)
$$

i.e. asymptotically $S(t) \leq(1-h(0))$. This in turn implies:

$$
I^{\prime} \leq \beta I\left(1-h(0)-R_{0}^{-1}\right)=\beta\left(p_{c}-h(0)\right) I .
$$

Thus, if $h(0)>p_{c}$ then $I(t) \rightarrow 0$ implying $p(t) \rightarrow h(0)$ and $S(t) \rightarrow 1-h(0)$, i.e. $E_{d f e}$ is GAS.

Let us now discuss the case of an exponentially fading memory in the perceived risk of vaccination with linear $h(I)=\vartheta I$. The Routh-Hurwitz stability condition reads:

$$
G(\Psi, b)=c_{2}(b) \Psi^{2}+c_{1}(b) \Psi+c_{0}(b)>0
$$


where $\Psi$ is as in eq. (17), and the coefficients are third order polynomials in the delay parameter $b$. Thus, for each $b$ there are at most two bifurcation values for $k$, whereas for each $k$ there may be at most three bifurcation values for $b$.

Acknowledgements We are greatly indebted to an anonymous referee for her/his suggestions that helped to improve this work significantly.

\section{References}

[1] Bauch, C. T., \& Earn, D. J. D., 2004 Vaccination and the theory of games. Proc. Natl. Acad. Sci. 101, 13391-13394.

[2] Bauch, C. T., 2005 Imitation dynamics predict vaccinating behaviour, Proc. R. Soc. B 272, 1669-1675.

[3] Brito, D.L., Sheshinski, E., \& Intriligator, M.D., 1991 Externalities and compulsory vaccinations. J. of Public Econ. 45,69-90.

[4] Center for Disease Control, Vaccine safety, http://www.cdc.gov/vaccinesafety/vaers/.

[5] Chen, R.T., \& Orenstein, W.A., 1996 Epidemiologic Methods in Immunization Programs. Epidemiologic Reviews 18 (2), 99-117.

[6] d'Onofrio, A., Manfredi, P., \& Salinelli, E., 2007 Vaccinating behaviour, information, and the dynamics of SIR vaccine preventable diseases. Th. Pop. Biol. 71, 301-317.

[7] d'Onofrio, A., Manfredi, P., \& Salinelli, E., 2008 Fatal SIR diseases and rational exemption to vaccination, Math. Med. and Biol., 25, 337-357.

[8] D'Onofrio, A., \& Manfredi, P. Information-related changes in contact patterns may trigger oscillations in the endemic prevalence of infectious diseases. J. Th. Biol. 259,473-478.

[9] d'Onofrio, A., \& Manfredi, P., 2010 Vaccine demand driven by vaccine side effects: dynamic implications for SIR diseases. J. Th. Biol. 264, 237-252

[10] Fine, P.E.M., \& Clarkson, J.A., 1986 Individual versus public priorities in the determination of optimal vaccination policies. Am. J. Epid. 124, 1012-1020.

[11] Galvani, A.P., Reluga, T.C., \& Chapman , G. B., 2007 Long-standing influenza vaccination policy is in accord with individual self-interest but not with the utilitarian optimum, PNAS 104, 5692-5697.

[12] Gangarosa, E.J., Galazka, A.M., Wolfe, C.R., Phillips, L.M., Gangarosa, R.E., Miller, E., \& Chen,R.T., 1998 Impact of anti-vaccine movements on pertussis control: the untold story, Lancet 351,356-61.

[13] Geoffard, P.Y., \& Philipson, T., 1997 Disease eradication: private versus public vaccination. Am. Econ. Rev. 87, 222-230.

[14] Hofbauer, J.,\& Sigmund, K., 1998 Evolutionary Games and Population Dynamics. Cambridge University Press, Cambridge, UK.

[15] Istituto Superiore di Sanità, Surveillance of adverse events, http://www.iss.it/sorveglianza/.

[16] Luman, E.T., Fiore, A.E., Strine, T.W., \& Barker, L.E., 2004 Impact of thimerosal-related changes in hepatitis B vaccine, birth-dose recommendations. JAMA 291, 2351-2358.

[17] Manfredi, P., della Posta, P., d'Onofrio, A., Salinelli, E., Centrone, F., Meo, C., \& Poletti, P., 2009 Optimal vaccination choice, vaccination games, and rational exemption: an appraisal, Vaccine 28, 98-109. 
[18] Molina, V., \& Shoenfeld, Y., 2005. Infection, vaccines and other environmental triggers of autoimmunity. Autoimmunity 38, 235-245.

[19] Perisic, A., \& Bauch, C.T., 2009 Social Contact Networks and Disease Eradicability under Voluntary Vaccination, PLoS Comp. Biol. 5, e1000280

[20] Roberts, R. J., Sandifer, Q. D., Evans, M.R., Nolan-Farrell, M.Z., \& Davis P.M. 1995 Reasons for non-uptake of measles, mumps and rubella catch up immunisation in a measles epidemic and side effects of the vaccine. Br. Med. J. 310,1629-1639.

[21] Reluga, T., Bauch, C., \& Galvani, A., 2006 Evolving public perceptions and stability in vaccine uptake. Math. Biosciences 204, 185-198.

[22] Reluga, T.C. 2009 An SIS epidemiology game with two subpopulations. J. Biol. Dyn. DOI: 10.1080/17513750802638399.

[23] Salmon, D.A., Teret, S.P., MacIntyre, C.R., Salisbury, D., Burgess, M.A., \& Halsey, N.A., 2006 Compulsory vaccination and conscientious or philosophical exemptions: past, present and future. Lancet 367, 436-442.

[24] Solomon, J., \& Murray, C.J.L., 2002 The epidemiological transition revisited: new compositional models for causes of death by age and sex. Pop \& Dev. Rev.28, 205-228.

[25] Efimov, D.V., \& Fradkov A.L. 2008 Yakubovich's oscillatority of circadian oscillations models, Math. Biosc. 216, 187-196.

[26] W.H.O. Immunization safety. hhttp://www.who.int/immunization_safety/aefi/en/i.

[27] W.H.O., 1999 Guidelines for managers of immunization programmes on reporting and investigating adverse events following immunization (WPRO/EPI/99.01.). Manila: WHO, Regional Office for the Western Pacific.
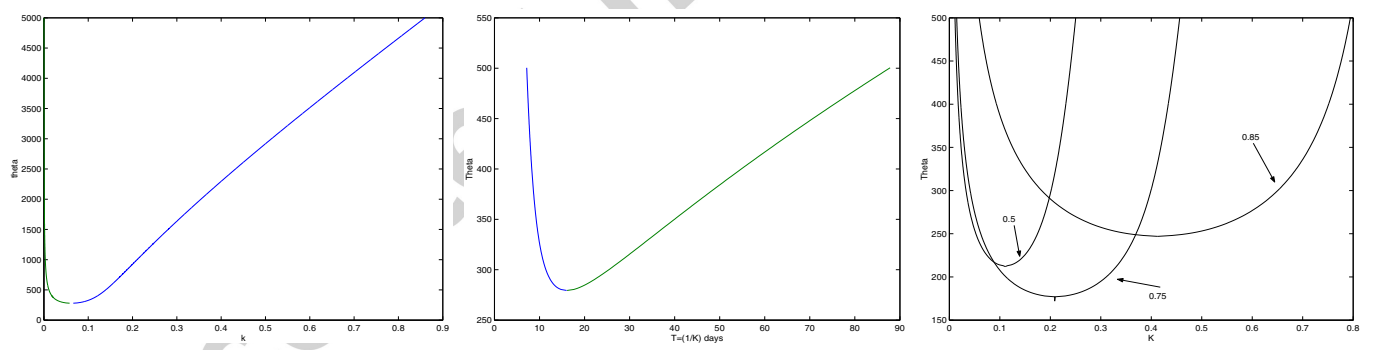

Figure 1: Bifurcation curve for linear and linear affine $h(I)$, in the case $R_{0}=10, \nu=1 / 10$ days $^{-1}, \mu=$ $1 / 50$ year $^{-1}$. (left-hand panel) Bifurcation curve for linear $h(I)=\vartheta I$ in the parameter space $(k, \vartheta)$. (Central panel) Bifurcation curve for linear $h(I)=\vartheta I$ in the parameter space $\left(k^{-1}, \vartheta\right)$ emphasizing patterns for small imitation-related delays. (Right-hand panel) Bifurcation curve for linear affine $h(I)=\vartheta_{0}+\vartheta I$ plotted for three values of $\vartheta_{0}$. As $\vartheta_{0}$ increases the minimal threshold of $\vartheta$ is nonmonotone. 

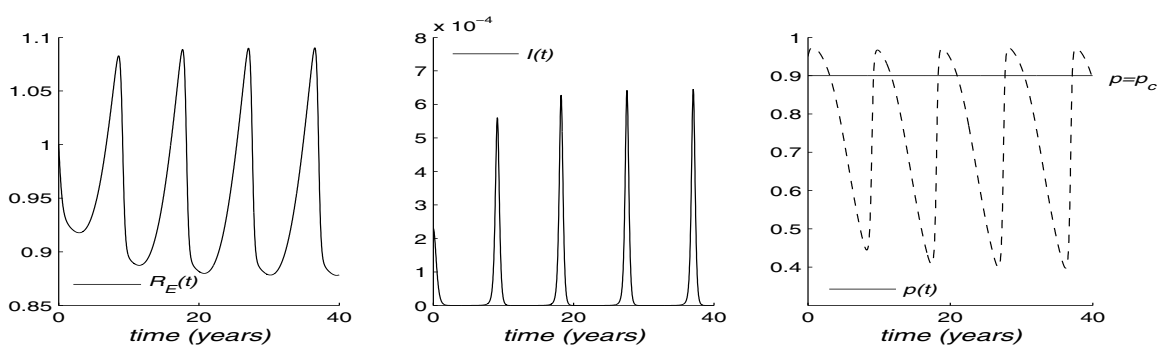

Figure 2: The unlagged model with linear $h(I)$ : dynamics of $R_{E}(t)$ (left-hand panel), $I(t)$ (centre), $p(t)$ (right-hand panel), following the initiation of an immunization programme with $p(0)=0.95$; $S_{0}, I_{0}$ fixed at their pre-vaccination endemic state. Parameters: $R_{0}=10, \nu=1 / 7$ days $^{-1}, \mu=1 / 75$ year $^{-1}, \vartheta=15000, k=0.002$ days $^{-1}$.

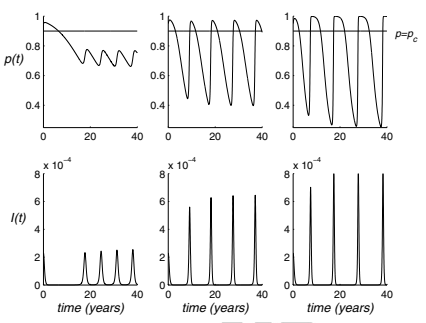

Figure 3: The unlagged model with linear $h(I)$ : dynamics of $p(t)$ (top) and $I(t)$ (bottom), for $k=$ 0.0005 days $^{-1}$ (left), $k=0.002$ days $^{-1}$ (centre), $k=0.0035$ days $^{-1}$ (right) following the initiation of an immunization programme with $p(0)=0.95 ; S_{0}, I_{0}$ fixed at their pre-vaccination endemic state. Other parameters: $R_{0}=10, \nu=1 / 7$ days $^{-1}, \mu=1 / 75$ year $^{-1}, \vartheta=15000$.
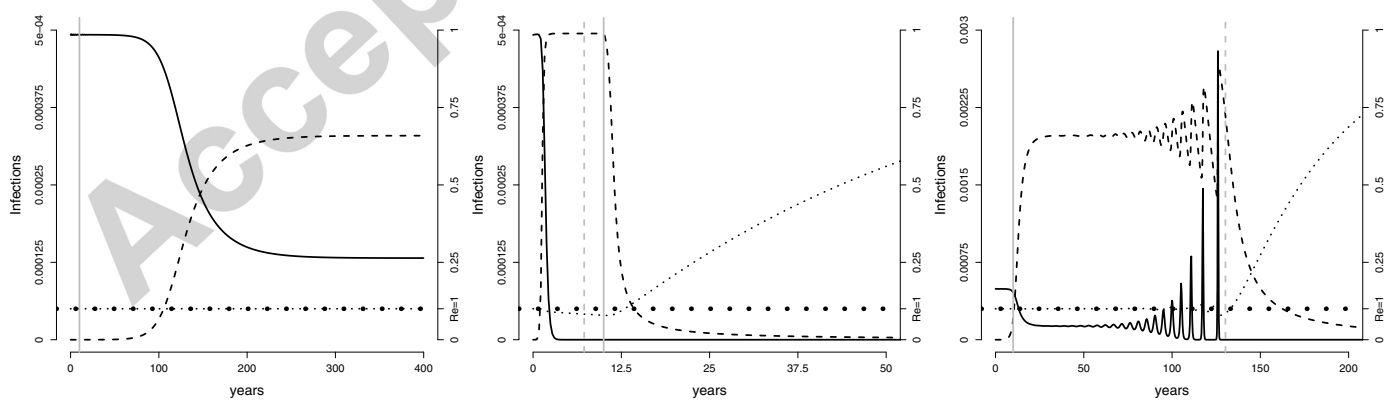

Figure 4: Dynamics of $I(t)$ (black line), $R_{0} S(t)$ (dotted black line), $p(t)$ (dashed black line) with (a), $k=0.0001$ days $^{-1}$ (b) and $k=0.001$ days $^{-1}$ (c). The vertical grey dashed line describe the time in which elimination occur. The vertical grey line describe the time in which VSE appears. Initial conditions: $S(0), I(0)$ fixed at pre-vaccination endemic state, $p(0) \simeq 0$. Other parameters: $R_{0}=10$, $\nu=1 / 10$ days $^{-1}, \mu=1 / 50$ year $^{-1}, \vartheta=5000$. 

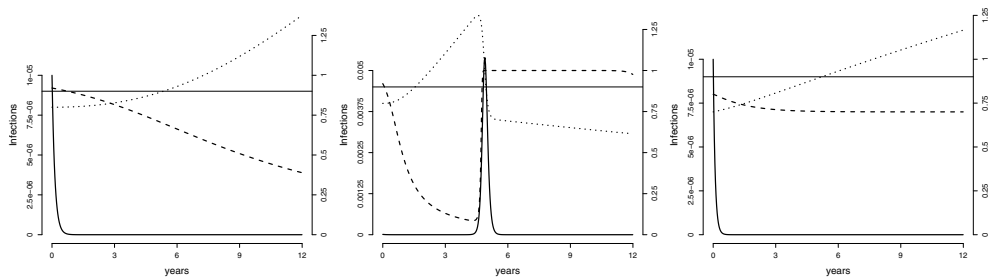

Figure 5: Left and central panels: dynamics of $I(t)$ (black line), $R_{0} S(t)$ (dotted black line), $p(t)$ (dashed black line) for $\vartheta=5000, \vartheta_{0}=0$ and $p(0)=0.95, S(0)=1-p(0), I(0)=10^{-5}$. Left-hand panel $k=0.001$ days $^{-1}$. Central panel: $k=0.01$ days $^{-1}$. The black line is the threshold $p_{c}$. Righthand panel: as the central panel but for $\vartheta_{0}=0.7, p(0)=0.80$ and $S(0)=0.07$. Other parameters: $R_{0}=10, \nu=1 / 10$ days $^{-1}, \mu=1 / 50$ years $^{-1}, \vartheta=5000$.
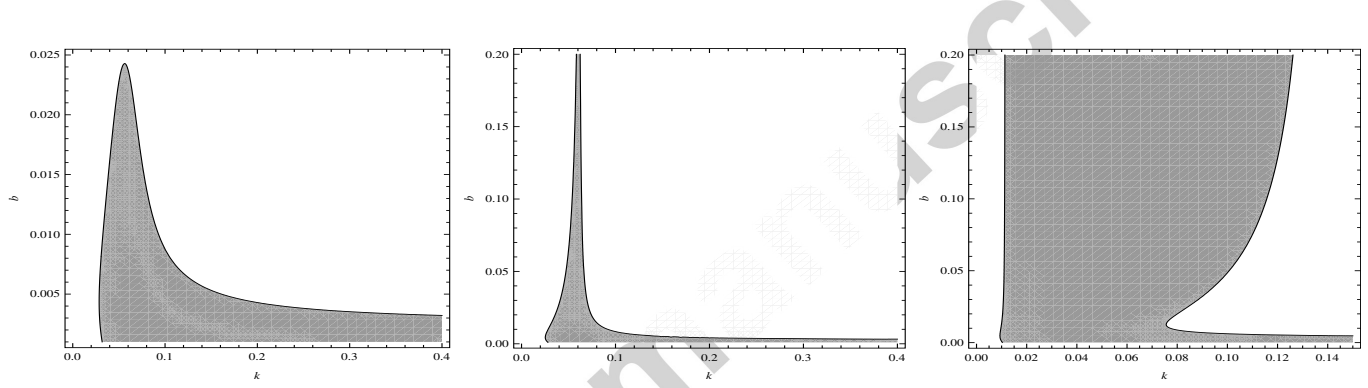

Figure 6: Effects of an exponentially fading memory in the perceived risk of infection: stability regions in the $(k, b)$ plane for $\vartheta=260$ (left), $\vartheta=500$ (right) and $\vartheta=279.5$ (centre), a value slightly higher than the bifurcation value. Other parameters: $R_{0}=10, \nu=1 / 10$ days $^{-1}, \mu=1 / 50$ years $^{-1}$.
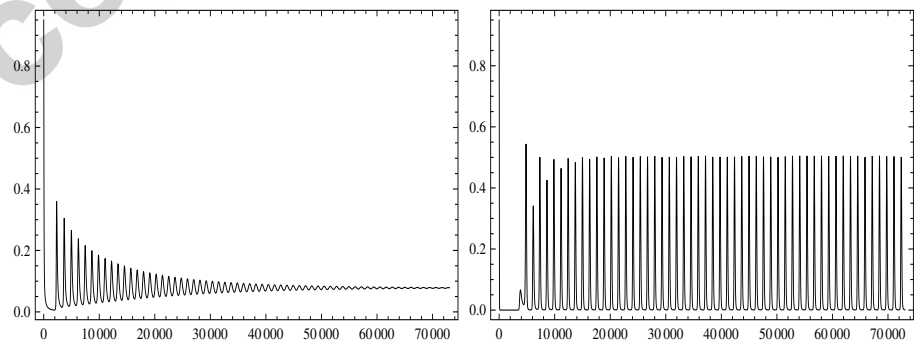

Figure 7: Effects of an exponential fading memory in the perceived risk of vaccination with $\vartheta=260$. Left-hand panel: the case of no delay. Right-hand panel: the delayed case with $b=0.004$ days $^{-1}$, corresponding to an average delay of 250 days. Other parameters: $R_{0}=10, \nu=1 / 10$ days $^{-1}$, $\mu=1 / 50$ years $^{-1}$. 

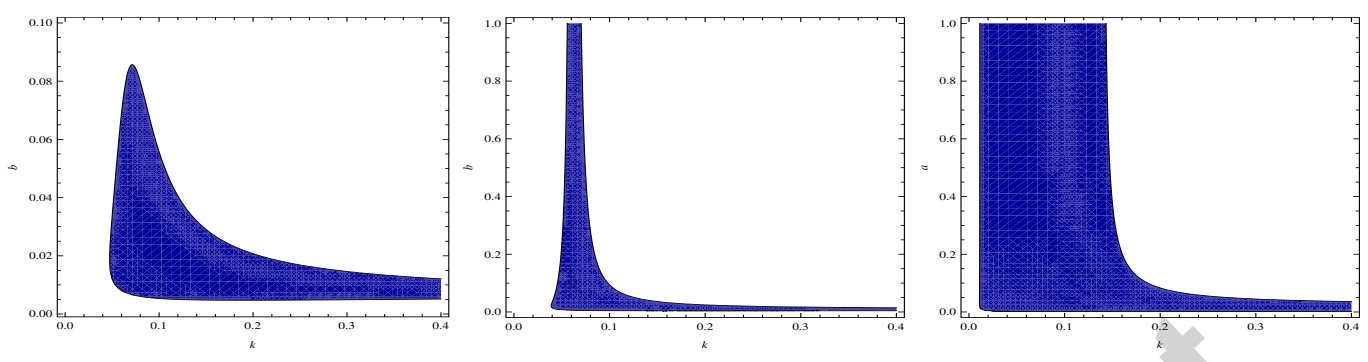

Figure 8: Information delayed case. Stability regions in the $(k, a)$ plane for $\vartheta=260$ (left), $\vartheta=500$ (right) and, in the centre, $\vartheta=279.5$, a value slightly higher than $\vartheta_{H}$. Other parameters: $R_{0}=10$, $\nu=1 / 10$ days $^{-1}, \mu=1 / 50$ years $^{-1}$. Note that in the central sub-figure the maximum $a$ is 100 .
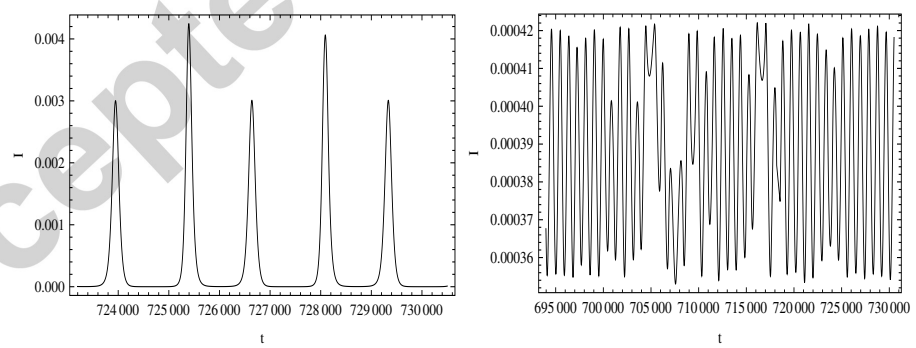

Figure 9: Effects on $I(t)$ of exponential fading memories in both information and side effects. In both panels $a=20 b, R_{0}=10, \nu=1 / 10$ days $^{-1}, \mu=1 / 50$ years $^{-1}$. (Left-hand panel) Periodic solution with alternating low and high peaks arising for $\vartheta=260, b=1 / 365.25$ days $^{-1}$; (Right-hand panel) Aperiodic solutions arising for $\vartheta=500, b=0.04$ days $^{-1}$. 